\title{
Cat Bite
}

National Cancer Institute

\section{Source}

National Cancer Institute. Cat Bite. NCI Thesaurus. Code C121560.

A wound received from the teeth of a cat. 